\title{
A Class-C Amplifier Linearized by a Constant Conduction Angle Biasing Circuit
}

\author{
Gursewak S. Rai, Vladimir Prodanov \\ Electrical Engineering Dept. \\ California Polytechnic State University - Cal Poly \\ San Luis Obispo, CA, USA
}

\begin{abstract}
This work presents a dynamic biasing topology capable of linearizing a Class-C power amplifier (PA). The topology utilizes a control loop that senses the operating conditions of the power device by means of a scaled replica. The loop operates on the principle of keeping the conduction angle constant and thereby ensuring linearity. The work details some of the design considerations that should prove useful to a designer wanting to implement the topology in an RF integrated circuit. Measurement results from a fully-functional lowfrequency prototype bring merit to the topology.
\end{abstract}

\section{INTRODUCTION}

Traditionally, class-C operation in power amplifiers has been avoided due to its inherent nonlinearity. However, stringent power control requirements in modern wireless communications (especially CDMA) have resulted in a renewed interest in class-C operation.

The interest in class- $\mathrm{C}$ operation is related to the fact that under power back-off conditions such amplifiers have improved efficiency as compared to Class-AB operation [1]. To overcome the problems of nonlinear distortion, several dynamic biasing schemes have been proposed [2-5]. The major issue in these topologies is their open-loop approach to dynamic biasing. Since the output may vary due to process variations and transistor thermal conditions, the performance of such circuits is not very robust.

In this paper, we present a topology that senses the output current of a scaled transistor replica and by the action of a closed loop, adjusts the gate bias of the power device in order to keep the conduction angle constant - thus ensuring linearity. This work builds upon others previously completed at Cal Poly under the supervision of V. Prodanov [6-7].

\section{The CONCEPT OF CONSTANT CONDUCTION-ANGLE BIASING}

Under sinusoidal input drive, the current of a transistor operated in a Class-B mode is ideally a half-wave rectified

\author{
Stephen Garber \\ Maxim Integrated Products \\ San Jose, CA, USA
}

sinusoid. The DC component and the fundamental component of the current are both linearly related to the magnitude of the input drive $\mathrm{V}_{\mathrm{AC}, \mathrm{B}}$.

$$
\begin{aligned}
& I_{D C, B}=\frac{1}{\pi} g_{m} V_{A C, B \mathrm{I}} \\
& I \mathrm{I}_{0, B}=\frac{1}{2} g_{m} V_{A C, B \mathrm{I}}
\end{aligned}
$$

Here $g_{m}$ denotes the slope of the transfer characteristic of the device. Slope is assumed to be either $\mathrm{g}_{\mathrm{m}}$ or zero.

The first expression shows that a device with an ideal piecewise linear V-I characteristic operated in Class-B mode can be used as a linear sensor of $\mathrm{V}_{\mathrm{AC}, \mathrm{B}}$. Expression (2) justifies the use of such a device in linear narrow-band amplifiers.

A transistor (with an ideal PWL V-I characteristic) can be used as a linear sensor and as a linear amplifier at any conduction angle $\theta$, as long as the conduction angle is kept constant. This is apparent from expressions (3) and (4).

$$
\begin{gathered}
I_{D C, \theta}=\frac{1}{\pi} g_{m}\left[\sin \frac{\theta}{2}-\frac{\theta}{2} \cos \frac{\theta}{2}\right] V_{A C, \theta} \\
I \mathrm{I}_{0, \theta}=\frac{1}{2 \pi} g_{m}[\theta-\sin \theta] V_{A C, \theta \mathrm{I}}
\end{gathered}
$$

The conceptual schematic of an RF power amplifier with constant-conduction-angle (CCA) bias generator is shown in Figure 1.

Transistors $\mathrm{M}_{\mathrm{B}}$ and $\mathrm{M}_{\mathrm{C}}$ are used as "sensors". The drain currents of these two devices are low-pass filtered and the low-frequency (DC) components are converted into voltages at the input of the error integrator.

The error integrator closes a feedback loop that dynamically adapts the gate bias of transistor $\mathrm{M}_{\mathrm{C}}$. This control scheme forces equal potentials at nodes $V_{B}$ and $V_{C}$ and establishes a constant ratio of DC currents [6].

$$
\frac{\mathrm{I}_{\mathrm{DC}, \mathrm{C}}}{\mathrm{I}_{\mathrm{DC}, \mathrm{B}}}=\frac{\mathrm{R}_{\mathrm{B}}}{\mathrm{R}_{\mathrm{C}}}=\text { const. I }
$$




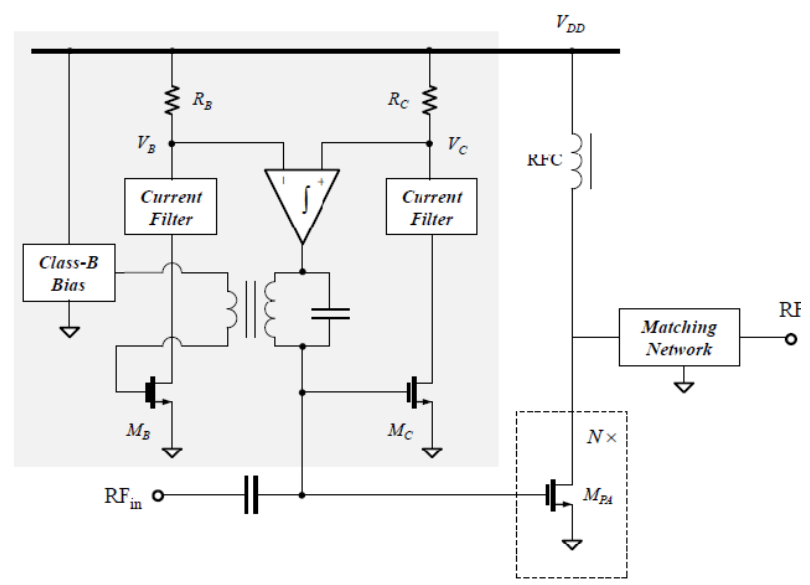

Figure 1. Concetual Schematic of a PA with CCA biasing

Unlike the approach presented in [6], here the Class-B sensor device is driven using a transformer. Thus, the input voltage drive presented to the Class-B device can be made different from that applied to the Class-C device. The drive ratio is fixed by the transformer turns ratio.

$$
\frac{\mathrm{V}_{\mathrm{AC}, \mathrm{B}}}{\mathrm{V}_{\mathrm{AC}, \mathrm{CI}}}=\text { const. I }
$$

Let us also assume that transistors $\mathrm{M}_{\mathrm{B}}$ and $\mathrm{M}_{\mathrm{C}}$ are identical. Then, by making use of (1), (3), (5) and (6) we can write the following relation:

$$
\frac{\mathrm{R}_{\mathrm{B}}}{\mathrm{R}_{\mathrm{C}}} \cdot \frac{\mathrm{V}_{\mathrm{AC}, \mathrm{B}}}{\mathrm{V}_{\mathrm{AC}, \mathrm{CI}}}=\sin \left(\frac{\theta}{2}\right)-\frac{\theta}{2} \cos \left(\frac{\theta}{2}\right)
$$

This equation indicates that the control loop will keep the conduction angle of transistor $\mathrm{M}_{\mathrm{C}}$ constant. The value of the conduction angle will be determined by the value of the lefthand side of equation (7). For a conduction angle less than $180^{\circ}$, the left-hand side of (7) must be less than unity.

Transistor $\mathrm{M}_{\mathrm{PA}}$ in Figure 1 is a physically-large device implemented as a parallel combination of many identical "unit" devices. Each individual device is assume to have exactly the same size as that of the sensor transistors $M_{B}$ and $\mathrm{M}_{\mathrm{C}}$. Transistor $\mathrm{M}_{\mathrm{PA}}$ together with the RF choke and the output matching network form a dynamically-biased RF PA.

\section{OPTIMIZED RATIOS}

The design of the proposed CCA biasing is an underconstrained problem, i.e. for a given conduction angle there are infinitely many combinations of resistor ratios and drive ratios that satisfy Equation (7). The ratios selected using relations (8) and (9) are of a particular interest because this set guarantees that $\mathrm{M}_{\mathrm{B}}$ and $\mathrm{M}_{\mathrm{C}}$ experience the same maximum gate overdrive [8].

$$
\left(\frac{\mathrm{R}_{\mathrm{B}}}{\mathrm{R}_{\mathrm{C}}}\right)_{\text {optI }}=\left(\frac{\mathrm{I}_{\mathrm{DC}, \mathrm{C}}}{\mathrm{I}_{\mathrm{DC}, \mathrm{B}}}\right)_{\text {optI }}=\frac{\sin \frac{\theta}{2}-\frac{\theta}{2} \cos \frac{\theta}{2} \mathrm{I}}{1-\cos \frac{\theta}{2}}
$$

$$
\left(\frac{\mathrm{V}_{\mathrm{AC}, \mathrm{B}}}{\mathrm{V}_{\mathrm{AC}, \mathrm{C}}}\right)_{\mathrm{optI}}=1-\cos \frac{\theta}{2}
$$

\section{A 1-MHz CONSTANT- $120^{\circ}$ PrototyPe}

To study the feasibility of the proposed approach a $1 \mathrm{MHz}$ demonstrator was implemented using discrete components.

The circuit schematic we implemented is shown in Figure 2. Photo of the implementation is included in Figure 3.

Three CA3086 arrays, each containing 5 npn devices, were used in the design. To improve the matching, all devices were emitter-degenerated using $22 \Omega$ resistors. The "power" device was constructed using twelve CA3086 devices. The other three CA3086 BJTs we used to create the fixed (Class-B) bias and the two "sensor" devices. The collector currents of the sensor transistors are low-pass filtered using 2-stage RC networks. The integrator is implemented using LM6132 railto-rail op-amp. The RC network at the output of the integrator is included to improve the gain margin of the loop. The control loop is stable and has a settling time of approximately $200-300 \mu \mathrm{sec}$. More details regarding the this particular topology can be found in [8].

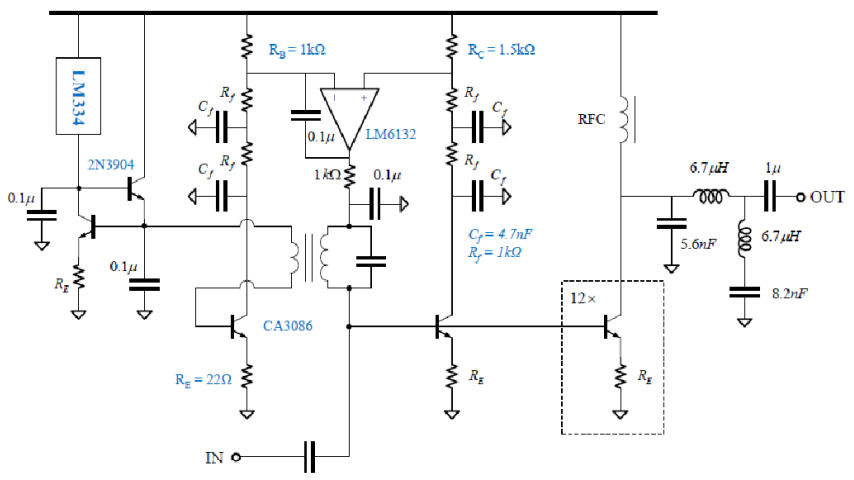

Figure 2. Complete Schematic of the Low-Frequency Prototype

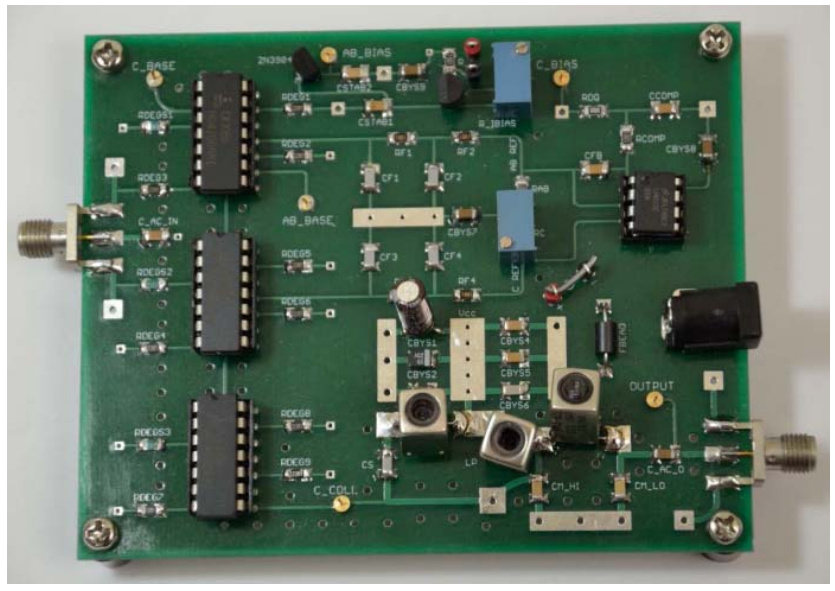

Figure 3. Photo of the Low-Frequency Prototype [8] 


\section{A. Experimantal Results}

Figure 4 shows that the input signals to the sensor transistors have approximately the same maximum excursions. This is expected because the optimum ratios, given in (8) and (9), were used in the design. The scope traces shown here correspond to a drive of $400 \mathrm{mVpeak}$. The equal peak excursion property was also verified for other input magnitudes.

Notice that the Class-B device is driven by a signal with smaller magnitude as necessitated by the desired conduction angle of $120^{\circ}$. The required 2-to-1 ratio is set by the transformer turns ratio. The transformer was constructed using 26 AWG (American Wire Gauge) magnetic wire on an FT3743 ferrite core.

Figure 5 shows that the conduction angle of the output current of the PA device is held constant over a range of input drives. Also notable is that the conduction interval is approximately $1 / 3$ of the period corresponding to a conduction angle of $120^{\circ}$ -as desired.

Consistent with the nearly-constant conduction angle is the reduction of DC biasing with the increase of input drive. This effect is demonstrated in Figure 6.

As third-order intermodulation distortion (IMD3) is an important figure of merit for an amplifier, a two-tone test was performed. Figure 7 shows the resulting IMD3 for a tone spacing of $500 \mathrm{~Hz}, 5 \mathrm{kHz}$ and $10 \mathrm{kHz}$.

The IMD3 characteristics seen in Figure 7 are consistent with theory - if the bandwidth of a signal is larger than the loop bandwidth, the control loop will not be able to effectively follow the envelope of the incoming signal and IMD3 will significantly increase.

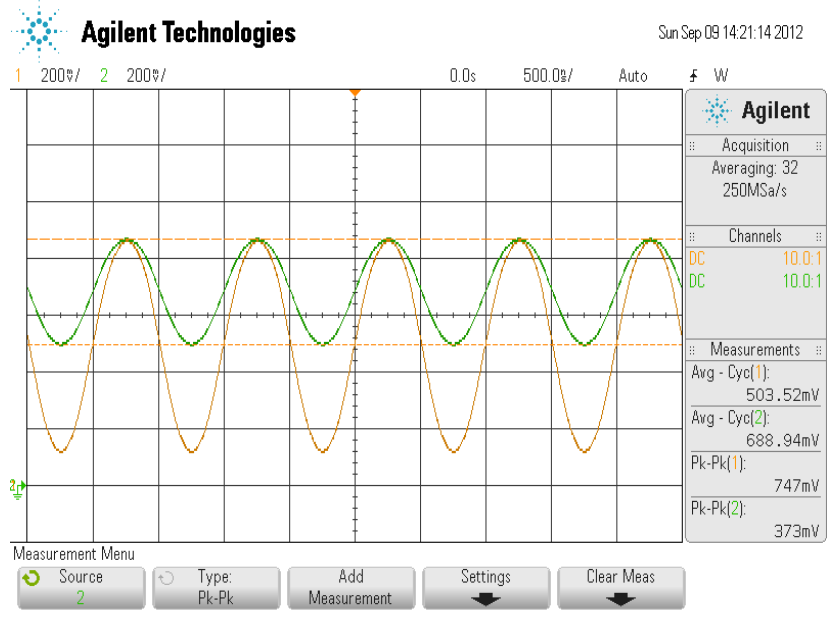

Figure 4. Equal peak excursions for Class-B and Class-C devices [8]
Agilent Technologies

Mon Sep 1007:28:24 2012

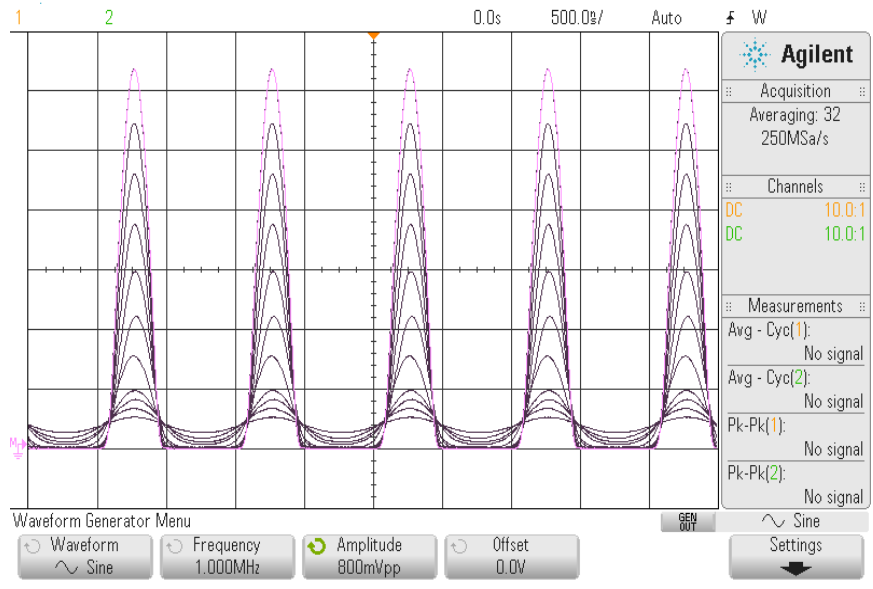

Figure 5. Voltage waveforms developing across a sense resistor connected in the collecter of the "power" device. [8]

\section{Base Biases of Class B Sensor \& C Sensor/PA}

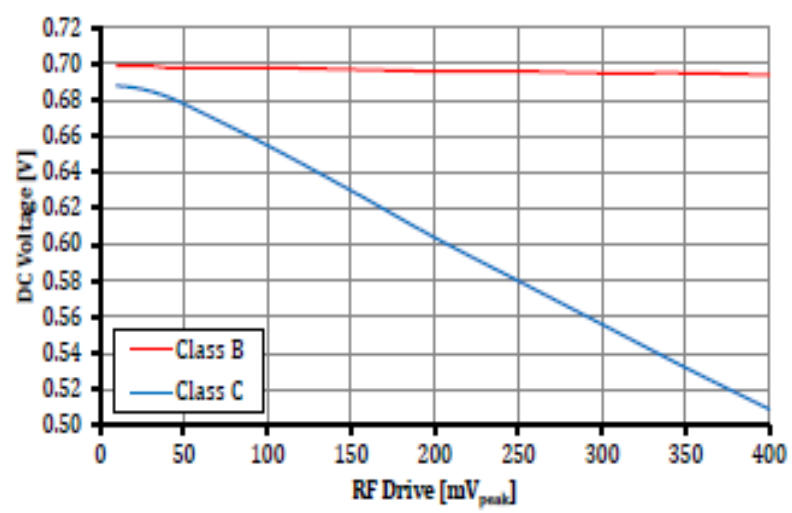

Figure 6. The DC base bias of the class C PA and the class B sensor [8]

$$
\mathrm{IMD}_{3} \text { of the Class C PA, } \mathrm{I}_{\mathrm{Bus}}=300 \mu \mathrm{A}
$$

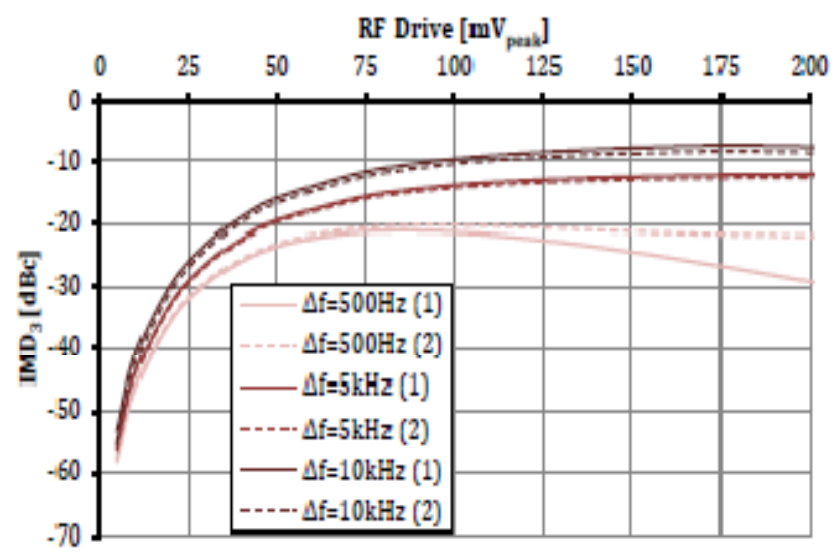

Figure 7. Measured Intermodulation Distortion for Various Input Drives and three different tone separations $(500 \mathrm{~Hz}, 5 \mathrm{kHz}$ and $10 \mathrm{kHz})[8]$ 


\section{SUMMARY AND CONCLUSSION}

We have presented a dynamic biasing arrangement that keeps the conduction angle of an amplifying device constant. The value of the conduction angle could be set smaller or larger than $180^{\circ}$. The operation of the circuit is based upon a simple theory which assumes a PWL transistor transfer characteristic - a condition that does not exist in practice. Despite that, a low-frequency prototype was successfully implemented and characterized. The test results demonstrate that the CCA biasing concept is a viable approach for linearization of Class-C amplifiers.

The next step in the evolution of the CCA biasing circuit is to implement the topology in a single IC at the intended cellular frequency.

\section{ACKNOWLEDGMENT}

The authors would like to acknowledge the contributions of Greg LaCaille. Mr. LaCaille was the first to investigate the properties of this particular dynamic biasing scheme [6].

\section{REFERENCES}

[1] V. I. Prodanov and M. Banu: 'Power Amplifier Principles and Modern Design Techniques'. In: Inewski, K. 'Wireless Technologies: Circuits, Systems, and Devices'. Boca Raton: CRC Press; 2008. pp. 349-389.

[2] T. Sowlati, 'Linearized Class C Amplifier with Dynamic Biasing'. United States of America Patent US 6,555,084 B2, 29 April 2003.

[3] G. Grillo and D. Cristaudo, 'Adaptive Biasing for UMTS Power Amplifiers', Bipolar/BiCMOS Circuits and Technology, 2004. Proceedings of the 2004 Meeting, 2004.

[4] S. Reed, Y. Wang, F. Huin, and S. Toutain, 'HBT Power Amplifier With Dynamic Base Biasing for 3G Handset Applications', Microwave and Wireless Components Letters, IEEE, vol. 14, no. 8, pp. 380-382, 2004.

[5] V. Leung, J. Deng, P. Gudem, and L. Larson, 'Analysis of Envelope Signal Injection for Improvement of RF Amplifier Intermodulation Distortion', IEEE J. Solid-State Circuits, vol. 40, no. 9, pp. 1888-1894, 2005.

[6] LaCaille, Greg: 'A Constant Conduction Angle Biased RF Power Amplifier for Improved Linearization in Class C Operation', MS thesis, Cal Poly, June 2010.

[7] Garber, Stephen: 'Constant Conduction Angle Bias Generation for Monolithic RF Power Amplifiers', Senior Design Report, Cal Poly, June 2011.

[8] Rai, Gursewak: 'Constant Conduction Angle Biasing for Class C Monolithic RF Power Amplifiers', MS Thesis, Cal Poly, Nov. 2012. 